\title{
Number of Renal Veins
}

National Cancer Institute

\section{Source}

National Cancer Institute. Number of Renal Veins. NCI Thesaurus. Code C130196.

The determination of the total number of renal veins. 